\title{
Arquitecturas de aspectos sociales en la práctica pedagógica de profesores en formación de lenguas extranjeras*
}

Architectures of Social Aspects in the Pedagogical Practice of Professors in Foreign Language Formation

Arquiteturas de aspectos sociais na prática pedagógica de professores em formação de línguas estrangeiras

\section{Harold Andrés Castañeda**}

Artículo de investigación

Revista Colombiana de Educación, N.7 75. Segundo semestre de 2018, Bogotá, Colombia.

doi: $10.17227 /$ rce.num75-8106

Para citar este artículo: Castañeda, H. (2018). Arquitecturas de aspectos sociales en la práctica pedagógica de profesores en formación de lenguas extranjeras. Revista Colombiana de Educación, 75, 139-158

\section{(c) $(1) \Theta$}

* Este artículo se deriva del proyecto "Eventos narrativos significativos de profesores en formación en torno a experiencias de práctica docente en la enseñanza de lenguas extranjeras: huellas desde la autovaloración", financiado por el Centro de Investigaciones y Desarrollo Científico (CIDC) de la Universidad Distrital Francisco José de Caldas, código 4-62-425-13, en el cual participaron investigadores de las universidades Distrital Francisco José de Caldas y Pontificia Universidad Javeriana.

** Doctor en Educación. Profesor Asociado Universidad Distrital Francisco José de Caldas, Bogotá, Colombia. Correo electrónico: hacastanedap@udistrital.edu.co 


\section{Resumen}

La investigación local sobre la práctica pedagógica de profesores en formación de lenguas extranjeras ha girado mayoritariamente en torno al papel de la reflexión. Este artículo de investigación propone que es necesario comprender las arquitecturas de práctica que se construyen socialmente en este contexto. El estudio se enfoca en la dimensión social de los modos de saber, hacer y de relacionarse de 184 participantes que diligenciaron un formato de autoevaluación de donde se extrajeron eventos narrativos. El análisis de los eventos narrativos se desarrolló, metodológicamente, desde la perspectiva de la teoría fundamentada. Los hallazgos ilustran tentativamente, entre otros, que una arquitectura plausible de práctica se manifiesta en la intersección de marcos de interpretación propios no siempre reconocidos en la literatura, en posicionamientos vehiculados a través de la mirada del otro y de sus disposiciones para enfrentar contextos diversos de enseñanza y en la acción conjunta aunada al logro de capital social del profesor en formación de lenguas extranjeras.

\section{Palabras clave}

arquitecturas de práctica; formación de docentes; modos de saber; modos de hacer; modos de relacionarse; lenguas extranjeras

\section{Keywords}

practice architectures; teacher training; ways of knowing; ways of doing; ways of relating; foreign languages

\begin{abstract}
Local research on the pedagogical practice of pre-service foreign language teachers has mostly revolved around the role of reflection. This research article suggests that it is necessary to understand the architectures of practice that are socially constructed in this context. The study focuses on the social dimension of the ways of knowing, doing and relating of 184 participants who completed a self-evaluation format from which narrative events were taken. The analysis of narrative events was methodologically developed from the perspective of grounded theory. The findings illustrate tentatively, among other things that a plausible architecture of practice is manifested in the intersection of their own interpretation frameworks not always recognized in the literature, in positions conveyed through the gaze of the other and their willingness to face diverse contexts of teaching and in the joint action together with the achievement of social capital of the pre-service foreign language teacher.
\end{abstract}

\section{Resumo}

A pesquisa local sobre a prática pedagógica de professores de línguas estrangeiras em formação tem se desenvolvido, principalmente, em torno à função da reflexão. Este artigo de pesquisa propõe que é preciso compreender as arquiteturas de prática que se constroem socialmente neste contexto. O estudo centra-se na dimensão social dos modos de saber, fazer e se relacionar de 184 participantes que preencheram o formato de autoavaliação do qual extraíram-se eventos narrativos. A análise desses eventos se desenvolveu, metodologicamente, desde a perspectiva da teoria fundamentada. As descobertas ilustram tentativamente, entre outras, que uma arquitetura plausível de prática se manifesta na intersecção de estruturas de interpretação próprias nem sempre reconhecidas na literatura, em posicionamentos veiculados através do olhar do outro e de suas disposições para enfrentar contextos diversos de ensino e na ação conjunta unificada à conquista de capital social do professor em formação de línguas estrangeiras.

\section{Palavras-chave}

arquiteturas de prática; formação de docentes; modos de saber; modos de fazer; modos de relacionar-se; línguas estrangeiras 


\section{Introducción}

La práctica pedagógica es el momento en el cual los profesores en formación de lenguas extranjeras despliegan sus conocimientos en un aula de clase en vivo y allí ponen en juicio sus criterios pedagógicos para realizar actos de educación (Richards y Nunan, 1990; Woodward, 1992). Ese momento, el de la práctica, puede ser un sitio epistemológico rico en información para que los programas de formación de docentes (re)piensen su espíritu formativo a partir de las experiencias vividas por los practicantes.

La relación entre lo brindado como educación para ejercer el magisterio y lo logrado al final del proceso merece atención por parte de los programas dedicados a formar futuros docentes para la sociedad. Hablamos entonces de la praxis educativa en el contexto de la educación de educadores, la cual ha sido un interés constante para los académicos colombianos y de otras latitudes que han formado generaciones de educadores en otros idiomas, entre los cuales predomina el inglés como lengua extranjera y minoritariamente se presenta el francés.

Sharky (2009) ve en la praxis educativa una transformación que vincula de manera dialógica la teoría y la práctica. Kemmis, Wilkinson, Edwards-Groves, Hardy, Grootenboer y Bristol (2014) ven más bien dos dimensiones. La primera se asume como lo instaurado en el halo de lo correcto; es decir, aquello que se debe enseñar porque está dictado por un compromiso institucional, o un currículo o un plan de estudios. Aquí la práctica de formación, de profesores de lenguas extranjeras, por ejemplo, está (pre)determinada por la tradición disciplinar y su fundamentación teórica. La segunda dimensión, que denominan como "una acción educativa que hace historia" (Kemmis et al., 2014, p. 26), devela más bien una ilusión por la resistencia a lo canónico (aunque se vive en la tradición) y se compromete con lo situado en los órdenes moral, social, ciudadano y, por qué no, humano. Para estos autores la educación es un proceso de iniciación en formas de comprender, de actuar y de relacionarse con el mundo para vivirlo (gozárselo) y coexistir en él: de apropiarlo como una práctica. Entonces, la práctica pedagógica de los profesores novicios de lenguas extranjeras parece escapar a una comprensión estática de la praxis como una operación directa de traducción de componentes teóricos (o de datos, conceptos, ideas, modelos, métodos de enseñanza) en procedimientos aplicados al aula donde se enseña un idioma extranjero. Por el contrario, se puede argumentar que se trata de la configuración de una arquitectura (de práctica) sobre la cual conocemos poco y sobre la cual interroga este artículo. 


\section{Exploraciones locales sobre la práctica pedagógica de profesores de inglés en formación}

Son varios los académicos colombianos que directamente se han ocupado de la práctica pedagógica en lenguas extranjeras como área de exploración (Cote, 2012; Insuasty y Zambrano, 2014; Prada y Zuleta, 2005; Viáfara, 2005a, 2005b, 2011; Zambrano e Insuasty, 2008, 2009; entre otros).

Se han creado espacios para que los estudiantes reflexionen sobre lo acontecido durante la práctica pedagógica y también se ha registrado cómo reflexionan para tomar acciones in situ; sin embargo, el nivel de reflexión apenas Ilega a estadios de criticidad (Cote, 2012). En esa dirección, pero en contextos de formación diferentes, Insuasty y Zambrano (2014) desarrollan el sistema de propuesta de experiencias reflexivas de asesoría (PERA), en tanto que la mayoría de profesores asesores del practicante tienden a ser prescriptivos e informativos. Es claro que

[...] la retroalimentación que ofrecen los asesores de práctica juega [sic] un papel importante en la formación inicial de los futuros docentes de inglés [y agregamos Francés], puesto que a través de ella se les orienta e informa sobre lo que saben, sobre lo que hacen y sobre su manera de actuar. (Insuasty y Zambrano, 2014, p. 409).

En ese hacer y manera de actuar, los profesores practicantes degustan "nuevos sabores" (Prada y Zuleta, 2005). Estos incluyen el uso de la lengua extranjera como medio de instrucción, de comunicación y como "objeto" de enseñanza (Viáfara, 2011); el manejo de la clase como continuo factor de observación e indicador de éxito; la selección de materiales tanto de enseñanza como de aprendizaje; la variedad de niveles de "proficiencia" presentes en un mismo salón de inglés o francés; entre muchos otros. Algunos de estos aspectos se vuelven instrumentales en la labor docente y comienza a perderse el ámbito educativo en el cual la enseñanza del idioma y sus actores están inmersos. No hay oportunidad de hacer historia en tanto se instaura lo canónico o correcto (Kemmis et al., 2014) como única posibilidad. Alternativas desde la literatura especializada emergen cuando se explora, por ejemplo, el uso de tareas de reflexión (Viáfara, 2005a; Zambrano e Insuasty, 2009) como una labor intrínseca a la práctica antes y después de experienciarla (Cote, 2012; Viáfara, 2005b; Zambrano e Insuasty, 2008). Sin embargo, la práctica pedagógica no es simplemente un lugar donde se da la reflexión personal sobre la experiencia con el ánimo de implementar estrategias de mejoramiento. Queremos argumentar que, sumado a esto, la práctica pedagógica de los profesores de lenguas extranjeras en formación en sí misma es un espacio que da lugar a la reflexión porque se constituye como práctica al poner en juego 
maneras de conocer, de hacer y de relacionarse; es decir, se trata de una arquitectura de práctica situada (Kemmis et al., 2014) que constituye un fenómeno del orden social.

\section{Arquitecturas de práctica}

Schatzki (2002) caracteriza el orden social como algo que es irregular-sea esta una característica del orden, por contradictorio que suene, hablamos de "otros" órdenes-, que da opción al sentido de lo inestable, es decir, que fluctúa y cambia y que acoge amplias gamas de (inter)relaciones entre las entidades que configuran ese orden. Es en esta idea -no canónica- de orden que puede verse la práctica pedagógica, o cualquier práctica, como una forma de "estar juntos", que al ser regularizada, se idealiza y se vuelve esencial: el orden social correcto. Sin embargo, la práctica pedagógica, vista como un orden social no canónico, se puede comprender como atravesada por el sentido de estar juntos en cuanto vínculo. Consecuentemente, el orden social es la (inter)relación de entidades mediante la existencia múltiple de nexos que nutren modos de saber y modos de hacer.

Kemmis et al. (2014) señalan que los modos de relacionarse son también formas de vínculo que forman parte de cualquier práctica. Son las múltiples posibilidades de vínculos entre estos tres modos los que (re) configuran diversas arquitecturas de prácticas (pedagógica(s) en lenguas extranjeras) en este caso particular. En sus palabras, la arquitectura de práctica se puede comprender como

[...] una forma de actividad humana cooperativa, establecida socialmente, en la que determinadas configuraciones de acciones y actividades (modos de hacer) son comprensibles en términos de configuraciones de ideas relevantes en discursos característicos (modos de saber) y cuando las personas y objetos involucrados se distribuyen en configuraciones de relaciones (modos de relacionarse) y cuando este complejo de modos de saber, de hacer y de relacionarse se articulan ("hang together") en un proyecto determinado. (Kemmins et al., 2014, p. 31).

El presente estudio examina esas formas de estar juntos en la práctica pedagógica desde la óptica de uno de sus actores: el profesor practicante de lenguas extranjeras. A partir de lo anterior se plantea como objetivo de este trabajo comprender las arquitecturas de práctica docente que los profesores de inglés y/o francés en formación conceptualizan, viven y experiencian en el momento de finalizar su práctica docente con el ánimo de responder cuáles manifestaciones de ella se construyen desde este marco de arquitecturas de práctica. 
En esta arquitectura de práctica (Kemmis et al., 2014), se entienden los modos de manera más precisa de la siguiente forma: los modos de saber constituyen un espacio de orden semántico en el cual los significados se (re)significan y negocian, se comprenden, se reciben y se reacciona a ellos de maneras específicas, de acuerdo con diversidad de intenciones y con el uso de modos del lenguaje (Rowsell y Walsh, 2015). Los modos de hacer constituyen un espacio de orden temporal donde se entretejen acciones y actividades que simplemente ocurren (re)acomodando los espacios en la medida en que los individuos asumen las actividades. Finalmente, un espacio de orden social se constituye al entrar en juego los modos de relacionarse. Estos develan cómo se conectan y relacionan los individuos entre sí, qué papeles asumen, sus relaciones formales e informales, el significado del poder, la confianza y la solidaridad y cómo se entienden los individuos en el espacio social.

\section{Método}

\section{Instrumento}

A través de un instrumento de autovaloración construido con los participantes se registraron eventos narrativos significativos (Chambers y Jurafsky, 2008; Griffin, 1993; Porter, Larson, Harthcock y Kelly, 2003) como unidad de análisis. Los participantes diligenciaron las autovaloraciones al final de su práctica pedagógica; estas giraron en torno a los espacios de formación brindados institucionalmente para dicho proceso: el lugar donde realizaron la práctica, el trabajo desarrollado con el mentor o guía pedagógico y el seminario de acompañamiento, que es una clase escolarizada.

\section{Participantes}

Se obtuvieron autovaloraciones de 184 participantes distribuidos en los periodos 2009-I, 2009-II, 2010-I, 2010-II, 2011-I, 2011-II, 2012-ı y 2012-II. El número de mujeres (75\%) triplicó el de hombres (25\%). Las edades de los participantes oscilaban entre los 19 y los 23 años en el momento de terminar su práctica pedagógica. El 90 \% de ellos realizaron su práctica pedagógica en colegios privados y el restante $10 \%$ en colegios de naturaleza estatal o distrital (públicos). El 80 \% realizó la práctica en inglés y el 20 \% en francés. Todos los participantes estaban cursando un programa de licenciatura en lenguas modernas en una universidad privada localizada en la capital del país. 

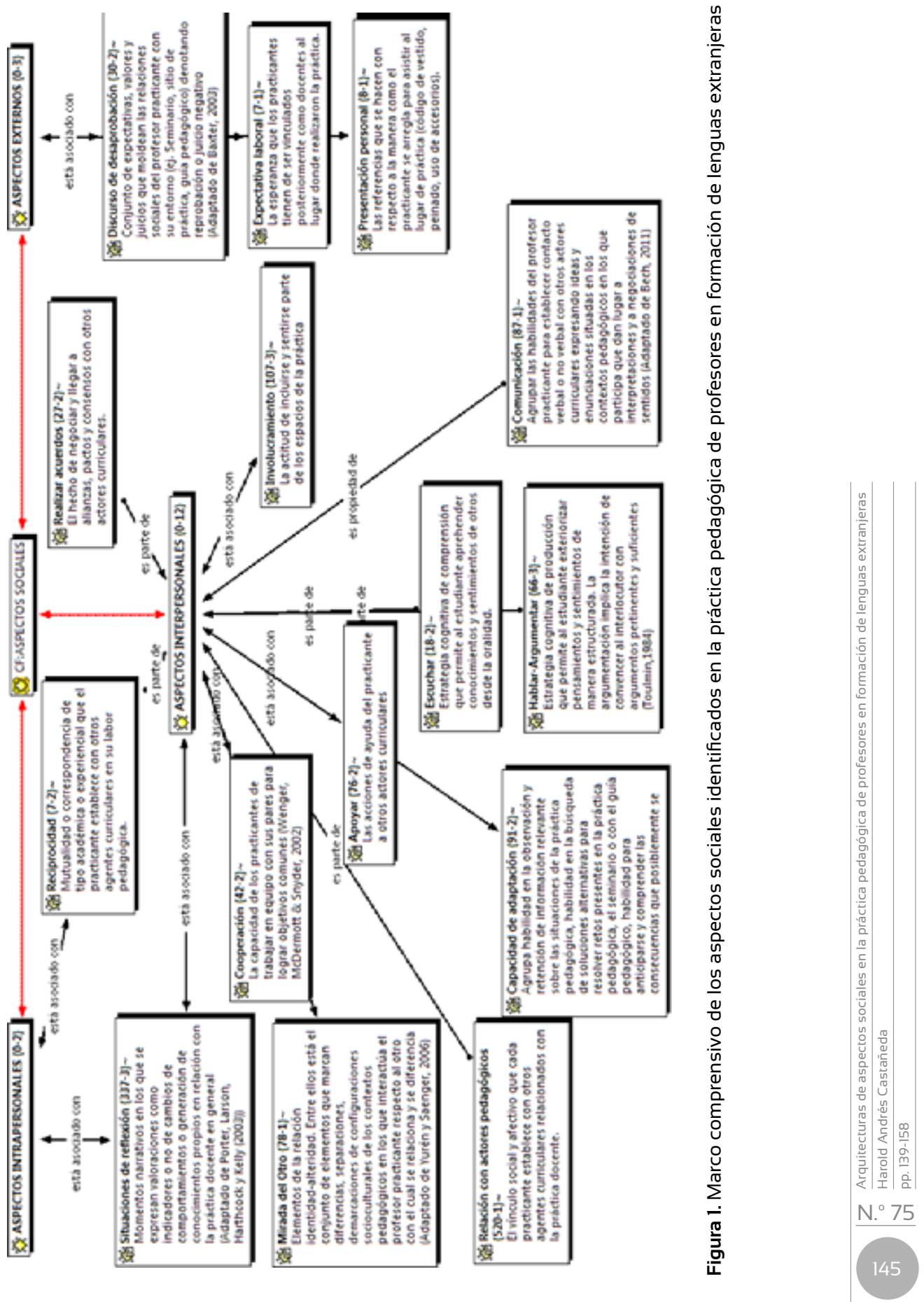


\section{Diseño}

El análisis de los eventos narrativos significativos se desarrolló metodológicamente desde la perspectiva de la teoría fundamentada (Strauss y Corbin, 2002). Se identificaron dimensiones precategoriales que incluyeron aspectos lingüísticos, sociales, cognitivo-emocionales y pedagógicos. Estas precategorías se codificaron utilizando un proceso iterativo entre todos los profesores investigadores del equipo a cargo del estudio; el resultado fue un marco comprensivo (véase la figura 1) y con validez configuracional para analizar la arquitectura de práctica pedagógica foco de indagación. En este trabajo hablaremos en particular de los aspectos sociales, siguiendo las categorías enunciadas en mayúscula en la figura 1.

\section{Resultados}

De acuerdo con el marco comprensivo descrito en la figura 1, hay tres rasgos para caracterizar la práctica pedagógica desde el ámbito de lo social: aspectos interpersonales, intrapersonales y externos. Estos se observan en los contextos mencionados: el sitio de práctica pedagógica o colegio, el seminario de apoyo a la formación inicial y el papel del guía pedagógico o mentor. Ejemplificaremos algunos casos seleccionados (45 citas para este artículo) $)^{1}$, que hemos sistematizado en la tabla 1, por la limitación de espacio. Para una discusión y ejemplificación más amplias se puede consultar Castañeda-Peña, Rodríguez-Uribe, Salazar-Sierra y Chala-Bejarano (2016a) y Castañeda-Peña et al. (2016b).

Tabla 1. Selección de ejemplos de aspectos sociales de arquitectura de práctica durante la práctica pedagógica de futuros profesores de lenguas extranjeras*

\begin{tabular}{|c|c|c|c|}
\hline Código & Colegio (1) & Seminario (II) & Guía pedagógico (II) \\
\hline $\begin{array}{c}\text { Apoyar } \\
\text { (interpersonal) }\end{array}$ & $\begin{array}{l}\text { Ol = Al colegio } \\
\text { asisto puntualmente } \\
\text { y cumplo con los } \\
\text { horarios establecidos, } \\
\text { además colaboro con } \\
\text { los profesores que } \\
\text { necesiten de mi ayuda, } \\
\text { los ayudo a calificar } \\
\text { exámenes (2009-॥) }\end{array}$ & $\begin{array}{l}\text { O2 = En cuanto al } \\
\text { proceso de mis } \\
\text { compañeros hubo } \\
\text { respeto y cuando } \\
\text { podíamos aconsejarle } \\
\text { o ayudarlo [sic] a } \\
\text { resolver problemas, } \\
\text { participé de } \\
\text { esto (2010-॥) }\end{array}$ & $\begin{array}{l}\text { O3 = Conté con ella } \\
\text { (la profesora guía } \\
\text { pedagógica) en } \\
\text { todo momento y } \\
\text { respondí con todos } \\
\text { los requisitos y } \\
\text { exigencias que ella y } \\
\text { yo acordamos (20ll-ı) }\end{array}$ \\
\hline
\end{tabular}

1 Para el aspecto social se codificaron de manera incluyente y abierta un total de 1501 citas dentro del corpus de eventos narrativos analizados, distribuidas en los 15 códigos ilustrados y definidos en la figura 1. Se incluye como muestra una selección de 45 citas en la tabla 1. 


\begin{tabular}{|c|c|c|c|}
\hline Código & Colegio (1) & Seminario (II) & Guía pedagógico (III) \\
\hline $\begin{array}{l}\text { Capacidad de } \\
\text { adaptación } \\
\text { (Interpersonales) }\end{array}$ & $\begin{array}{l}\text { O4 = Asimismo, } \\
\text { siempre respeté las } \\
\text { normas del colegio } \\
\text { como a las profesoras, } \\
\text { psicólogas y demás } \\
\text { personal de la } \\
\text { institución (2010-I) }\end{array}$ & $\mathrm{O} 5=\mathrm{NA}$ & $\begin{array}{l}\text { O6 = ... siempre } \\
\text { nos comunicaron } \\
\text { (sic) para acordar } \\
\text { nuestros encuentros } \\
\text { a los cuales asistí } \\
\text { puntualmente (2012-I) }\end{array}$ \\
\hline $\begin{array}{c}\text { Comunicación } \\
\text { (Interpersonales) }\end{array}$ & $\begin{array}{l}\text { O7 = Los estudiantes } \\
\text { expresaban con gran } \\
\text { alegría su agrado } \\
\text { de que yo fuera su } \\
\text { maestro (2009-॥) }\end{array}$ & $\begin{array}{l}\text { O8 = Tengo una buena } \\
\text { relación dialógica } \\
\text { con ambos (profesor } \\
\text { del seminario y } \\
\text { guía pedagógico) } \\
\text { lo que me permitió } \\
\text { entender y aceptar } \\
\text { la retroalimentación } \\
\text { y trabajar en esos } \\
\text { aspectos para } \\
\text { mejorar (2012-ı) }\end{array}$ & $\begin{array}{l}\text { O9 = ... en general el } \\
\text { compromiso con la } \\
\text { guía fue bueno y se } \\
\text { presentó una buena } \\
\text { comunicación (2009-॥) }\end{array}$ \\
\hline $\begin{array}{c}\text { Cooperación } \\
\text { (Interpersonales) }\end{array}$ & $\begin{array}{l}\text { OlO = [Nombre } \\
\text { profesor del colegio] } \\
\text { depositó confianza en } \\
\text { mí y pidió mi opinión } \\
\text { e ideas varias veces } \\
\text { para el desarrollo } \\
\text { de la clase (2011-ı) }\end{array}$ & $\begin{array}{l}\text { Oll = ... las clases } \\
\text { me gustaron mucho } \\
\text { porque podíamos } \\
\text { ayudarnos con } \\
\text { situaciones en el } \\
\text { colegio y recibir } \\
\text { varios consejos } \\
\text { antes de decidir qué } \\
\text { hacer (2010-॥) }\end{array}$ & $\begin{array}{l}\text { O12 = ... el trabajo con } \\
\text { el guía pedagógico } \\
\text { generó importantes } \\
\text { retroalimentaciones } \\
\text { que enriquecieron mi } \\
\text { práctica profesional, y } \\
\text { considero que también } \\
\text { aporté algunos } \\
\text { conocimientos } \\
\text { pedagógicos } \\
\text { didácticos a mi guía, } \\
\text { los cuales plantearon } \\
\text { discusiones muy } \\
\text { productivas a nivel } \\
\text { profesional (2012-ı) }\end{array}$ \\
\hline $\begin{array}{c}\text { Escuchar } \\
\text { (Interpersonales) }\end{array}$ & $\begin{array}{l}\text { Ol3 = Mi relación } \\
\text { con las profesoras } \\
\text { del departamento } \\
\text { me ayudó a ver otro } \\
\text { punto de vista más } \\
\text { amplio tanto a nivel } \\
\text { educativo como } \\
\text { laboral, siempre estuve } \\
\text { abierta a sus consejos } \\
\text { y recomendaciones } \\
\text { (2011-ı) }\end{array}$ & $\begin{array}{l}\text { O14 = ... soy consciente } \\
\text { [de] que mi fortaleza } \\
\text { no era participar en } \\
\text { clase no porque no } \\
\text { supiera qué decir } \\
\text { sino porque mi } \\
\text { estilo es escuchar } \\
\text { y tomar apuntes en } \\
\text { clase (2009-॥) }\end{array}$ & $\begin{array}{l}\text { Ol5 = Escuché y } \\
\text { puse en práctica el } \\
\text { feedback que ella } \\
\text { me daba (2012-॥) }\end{array}$ \\
\hline $\begin{array}{c}\text { Hablar } \\
\text { (Interpersonales) }\end{array}$ & $\begin{array}{l}\text { Ol6 = Con profesores } \\
\text { de otras áreas, } \\
\text { pude comentar } \\
\text { el rendimiento y } \\
\text { comportamiento de } \\
\text { los estudiantes; si } \\
\text { era igual en todas las } \\
\text { materias (2010-॥) }\end{array}$ & $\begin{array}{l}\text { Ol7 = Participé } \\
\text { constantemente en } \\
\text { el seminario y lo hice } \\
\text { con aportes válidos y } \\
\text { sobre lo que se estaba } \\
\text { hablando; es decir, } \\
\text { fui relevante en mis } \\
\text { opiniones en todo } \\
\text { momento (2011-ı) }\end{array}$ & $\begin{array}{l}\text { Ol8 = ... nos reuníamos, } \\
\text { y discutíamos el tema } \\
\text { y el proceso que se } \\
\text { iba a llevar a cabo } \\
\text { en la clase (2010-॥) }\end{array}$ \\
\hline
\end{tabular}




\begin{tabular}{|c|c|c|c|}
\hline Código & Colegio (1) & Seminario (II) & Guía pedagógico (III) \\
\hline $\begin{array}{l}\text { Involucramiento } \\
\text { (Interpersonales) }\end{array}$ & $\begin{array}{l}\text { O19 = Me encanta } \\
\text { estar en el colegio } \\
\text { por eso no solo he } \\
\text { observado a las } \\
\text { clases de inglés [sic] } \\
\text { también he observado } \\
\text { todo el ambiente ... } \\
\text { en jardín, primaria y } \\
\text { bachillerato (2009-ı) }\end{array}$ & $\mathrm{O} 20$ = na & $\mathrm{O} 21=\mathrm{NA}$ \\
\hline $\begin{array}{l}\text { Mirada del Otro } \\
\text { (Interpersonales) }\end{array}$ & $\begin{array}{l}\text { O22 = Los profesores } \\
\text { estuvieron muy } \\
\text { pendientes de mi } \\
\text { desempeño como } \\
\text { docente, como } \\
\text { amiga de los niños } \\
\text { y como huésped } \\
\text { del colegio, así me } \\
\text { hicieron sentir (2Oll-I) }\end{array}$ & $\begin{array}{l}\text { O23 = Considero } \\
\text { que en el seminario } \\
\text { mi participación fue } \\
\text { acertada y apreciada } \\
\text { por los compañeros, } \\
\text { en tanto siempre } \\
\text { estuve dispuesta a dar } \\
\text { mi opinión que podría } \\
\text { aportarle a la discusión } \\
\text { de la clase (2011-ı) }\end{array}$ & $\begin{array}{l}\text { O24 = Al sentir } \\
\text { que tanto mi guía } \\
\text { pedagógica como } \\
\text { mi Profesor titular } \\
\text { desarrollaron una } \\
\text { paulatina confianza } \\
\text { en mí fue una } \\
\text { responsabilidad y } \\
\text { un incentivo para } \\
\text { mantener mi perfil } \\
\text { siempre activo y } \\
\text { dispuesto a las } \\
\text { varias labores de la } \\
\text { enseñanza (20ll-॥) }\end{array}$ \\
\hline $\begin{array}{c}\text { Realizar } \\
\text { acuerdos } \\
\text { (Interpersonales) }\end{array}$ & $\begin{array}{l}\text { O25 = Aunque al } \\
\text { principio para las } \\
\text { profesoras era difícil } \\
\text { hacerse a la idea de } \\
\text { que podían solicitar } \\
\text { mi ayuda, en cualquier } \\
\text { caso, en el último } \\
\text { mes se logró llegar } \\
\text { a un acuerdo con } \\
\text { ellas, ya que durante } \\
\text { este tiempo ya no } \\
\text { se estaban dictando } \\
\text { clases (2009-॥) }\end{array}$ & $026=N A$ & $\begin{array}{l}\text { O27 = Las } \\
\text { observaciones } \\
\text { también fueron } \\
\text { acordadas con } \\
\text { anterioridad por ambas } \\
\text { partes y hubo un } \\
\text { trabajo en equipo con } \\
\text { los otros practicantes } \\
\text { para que la guía solo } \\
\text { tuviera que asistir } \\
\text { un día (2Oll-ı) }\end{array}$ \\
\hline $\begin{array}{c}\text { Reciprocidad } \\
\text { (Interpersonales, } \\
\text { intrapersonales) }\end{array}$ & $\begin{array}{l}\text { O28 = Me encantó } \\
\text { que cuando no } \\
\text { sabía la respuesta } \\
\text { de algo entre todos } \\
\text { analizamos el tema en } \\
\text { cuestión y llegamos } \\
\text { a una conclusión, se } \\
\text { generó un ambiente } \\
\text { de aprendizaje } \\
\text { mutuo. Mis alumnos } \\
\text { me enseñaron } \\
\text { muchas cosas, y me } \\
\text { aconsejaron cómo } \\
\text { mejorar algunas } \\
\text { cosas (2012-ı) }\end{array}$ & $\begin{array}{l}\text { O29 = Los foros fueron } \\
\text { interesantes porque } \\
\text { con la respuesta de los } \\
\text { demás compañeros } \\
\text { se podía tener varias } \\
\text { perspectivas acerca } \\
\text { de las situaciones } \\
\text { que se planteaban } \\
\text { para discutir (2010-ı) }\end{array}$ & $\begin{array}{l}\text { O30 = ... el trabajo con } \\
\text { el guía pedagógico } \\
\text { generó importantes } \\
\text { retroalimentaciones } \\
\text { que enriquecieron mi } \\
\text { práctica profesional, y } \\
\text { considero que también } \\
\text { aporté algunos } \\
\text { conocimientos } \\
\text { pedagógicos } \\
\text { didácticos a mi guía, } \\
\text { los cuales plantearon } \\
\text { discusiones muy } \\
\text { productivas a nivel } \\
\text { profesional (2012-ı) }\end{array}$ \\
\hline
\end{tabular}




\begin{tabular}{|c|c|c|c|}
\hline Código & Colegio (1) & Seminario (II) & Guía pedagógico (III) \\
\hline $\begin{array}{c}\text { Relación con } \\
\text { actores } \\
\text { (Interpersonales) }\end{array}$ & $\begin{array}{l}\text { O31 = Con todas las } \\
\text { profesoras hay una } \\
\text { buena relación, no solo } \\
\text { ayudo a mi profesora } \\
\text { titular sino a las demás } \\
\text { también }(2011-1)\end{array}$ & $\begin{array}{l}\text { O32 = Respeté el } \\
\text { proceso de cada uno } \\
\text { de mis compañeros, } \\
\text { pues todos estamos } \\
\text { en las mismas } \\
\text { condiciones (2010-॥) }\end{array}$ & $\begin{array}{l}\text { O33 = ... con un gran } \\
\text { ser humano que me } \\
\text { acompañó, guió, criticó, } \\
\text { exaltó y sugirió el } \\
\text { modo en que podría } \\
\text { desempeñarme en } \\
\text { el colegio y con los } \\
\text { demás (2010-ı) }\end{array}$ \\
\hline $\begin{array}{c}\text { Situaciones } \\
\text { de reflexión } \\
\text { (Intrapersonales, } \\
\text { interpersonales) }\end{array}$ & $\begin{array}{l}\text { O34 = Estas clases } \\
\text { fueron para mí } \\
\text { verdaderamente } \\
\text { útiles y de total } \\
\text { aprovechamiento ya } \\
\text { que me permitían } \\
\text { caer en cuenta de } \\
\text { aquellas debilidades } \\
\text { para así mejorarlas } \\
\text { en las siguientes } \\
\text { clases (2011-ı) }\end{array}$ & $\begin{array}{l}\text { O35 = Lo que más } \\
\text { gustó, y en lo que } \\
\text { considero tuve un } \\
\text { mejor desempeño fue } \\
\text { en las ocasiones en } \\
\text { que todos hacíamos } \\
\text { discusiones acerca } \\
\text { de las diferentes } \\
\text { experiencias que } \\
\text { íbamos teniendo } \\
\text { en los diferentes } \\
\text { colegios (2010-॥) }\end{array}$ & $\begin{array}{l}\text { O36 = Durante las } \\
\text { actividades realizadas } \\
\text { en el colegio } \\
\text { procuré integrar las } \\
\text { observaciones y } \\
\text { sugerencias dadas por } \\
\text { mi guía pedagógica } \\
\text { y reflexionar sobre } \\
\text { mi desempeño y } \\
\text { los resultados al } \\
\text { hacerlo (2009-॥) }\end{array}$ \\
\hline $\begin{array}{l}\text { Presentación } \\
\text { personal } \\
\text { (Externos) }\end{array}$ & $\begin{array}{l}\text { O37 = Además, } \\
\text { siempre me esmeré } \\
\text { por estar bien } \\
\text { arreglado y puntual } \\
\text { para iniciar la reflexión } \\
\text { en el colegio (2011-ı) }\end{array}$ & $\mathrm{O} 38$ = NA & $\mathrm{O} 39=\mathrm{NA}$ \\
\hline $\begin{array}{l}\text { Expectativa } \\
\text { laboral } \\
\text { (Externos) }\end{array}$ & $\begin{array}{l}\text { O40 = El colegio } \\
\text { [nombre de colegio] } \\
\text { me abrió las puertas } \\
\text { para ser parte del } \\
\text { mismo y dejar } \\
\text { una huella en los } \\
\text { estudiantes de } \\
\text { primaria (2011-ı) }\end{array}$ & $\mathrm{O} 41=\mathrm{NA}$ & $\begin{array}{l}\text { O42 = ... el } \\
\text { acompañamiento } \\
\text { es bueno en el } \\
\text { sentido de que otra } \\
\text { persona que da una } \\
\text { retroalimentación } \\
\text { para futuros } \\
\text { trabajos (2010-ı) }\end{array}$ \\
\hline $\begin{array}{c}\text { Discurso de } \\
\text { desaprobación } \\
\text { (Externos) }\end{array}$ & $\begin{array}{l}\text { O43 = Por otro lado, } \\
\text { no comparto muchas } \\
\text { cosas de la filosofía de } \\
\text { la institución los cuales } \\
\text { [sic] en ocasiones } \\
\text { modificaban mi } \\
\text { comportamiento en } \\
\text { el aula y en la misma } \\
\text { institución, el hecho } \\
\text { de escuchar a los } \\
\text { docentes decir que no } \\
\text { hay esperanza con los } \\
\text { muchachos, desanima } \\
\text { mucho (2011-॥) }\end{array}$ & $\begin{array}{l}\text { O44 = Desde mi } \\
\text { punto de vista el } \\
\text { seminario fue un poco } \\
\text { denso (2009-॥) }\end{array}$ & $\begin{array}{l}\text { O45 = Los planes de } \\
\text { clase eran muchas } \\
\text { veces imposibles } \\
\text { de entregar, debido } \\
\text { a que en el colegio } \\
\text { debíamos improvisar } \\
\text { las clases, por lo tanto, } \\
\text { no fueron muchas las } \\
\text { entregas y además } \\
\text { la retroalimentación } \\
\text { era a veces imposible } \\
\text { de poner en práctica } \\
\text { debido a que la } \\
\text { entrega y la revisión } \\
\text { y el desarrollo de la } \\
\text { clase se daban de un } \\
\text { día para otro (2009-॥) }\end{array}$ \\
\hline
\end{tabular}

* La tabla muestra en la primera columna los códigos abiertos que realizan las tres categorías principales. Debajo de los nombres de los códigos se señala su relación lógica con las categorías entre paréntesis, en concordancia con el marco comprensivo de la figura 1. En sentido horizontal se puede leer un aparte del evento narrativo seleccionado de cada código (enumerados (1), (2), ...) en tres contextos observados: sitio de práctica pedagógica o colegio (I), el seminario de apoyo a la formación inicial (II) y el 
papel del guía pedagógico o mentor (III). Luego de cada ejemplo se observa entre paréntesis la cohorte de la cual proviene el evento narrativo. Al referirnos a los ejemplos, en el texto de análisis y discusión, utilizamos la convención (1-01-2009-॥) en donde I = rasgo, en este caso interpersonal, $\mathrm{Ol}$ = ejemplo de evento narrativo y 2009-ı = cohorte de donde proviene el ejemplo.

\section{Rasgos sociales interpersonales, intrapersonales y externos en el colegio}

Desde lo interpersonal, el colegio se convierte en un espacio físico y cultural en donde compartir saberes con los actores curriculares, especialmente profesores titulares y estudiantes, gana un peso importante. Esto se evidencia, por ejemplo, en la reciprocidad del apoyo que puede darse en un sentido técnico de adaptación al nuevo sitio de práctica como el calificar exámenes (I-01-2009-II). Este es un aprendizaje en el cual se comparte saber cómo hacer acciones naturalizadas en el ejercicio docente y en el cual se tejen socialmente lazos de confianza, donde se negocian sentidos, al menos con relación a la opinión (I-010-2011-I; I-013-2011-I) y la puesta en marcha de los saberes (I-022-2011-I; I-031-2011-I). El saber se fortalece al entender rasgos propios de la cultura escolar y apropiarlos (I-04-2010-ו) y al comprenderse como actor que es reconocido por lo que sabe y que se posiciona de la misma forma: "los estudiantes expresaban con gran alegría su agrado de que yo fuera su maestro" (I-07-2099-II). Este rasgo también se vio fortalecido al discutir la escuela con docentes de otras áreas o al examinar otros niveles de formación (I-016-2010-II; I-0192009-II). Sin embargo, participar en la construcción del conocimiento no es fácil ya que se perciben resistencias (I-025-2009-ı); lo importante en lo interpersonal parece ser que el conocimiento traído por el profesor en formación se reta, quizá valida, con el que encuentra en los demás actores que comparten el espacio escolar y pasa por momentos de consolidación y de transformación vinculados a maneras de interactuar y de hacer.

Este último aspecto se ve reflejado en la experiencia de un profesor practicante cuando cae en la cuenta de que su conocimiento no es total o estático y que, al intentar expresarlo en su práctica en el aula, este se puede fortalecer con el conocimiento que aportan sus estudiantes: "Me encantó que cuando no sabía la respuesta de algo entre todos analizamos el tema en cuestión y llegamos a una conclusión, se generó un ambiente de aprendizaje mutuo" (I-028-2012-I). El saber es entonces negociado y no transmitido. Se percibe de esa manera un modelo de aprendizaje cooperado -quizá constructivista- y no transmisionista o bancario. Además, el performance docente, o sus otras maneras de hacer las cosas en el espacio de práctica, comporta la retroalimentación para el mejoramiento y la innovación en la misma práctica: "Mis alumnos me enseñaron muchas cosas, y me aconsejaron cómo mejorar algunas cosas" (I-028-2012-I). Esto ilustra un aspecto 
de innovación frente al modelo tradicionalmente instaurado, al menos en la práctica pedagógica de lenguas extranjeras, en que la reflexión intrapersonal se propicia para simplemente "mejorar" en el aspecto instruccional: "Estas clases fueron para mi verdaderamente útiles y de total aprovechamiento ya que me permitían caer en cuenta de aquellas debilidades para así mejorarlas en las siguientes clases" (I-034-2011-I). La (co)construcción del saber hacer en el aula y del saber en cuanto a contenido genera unos modos de saber que son poco reconocidos en la literatura clásica de la práctica pedagógica en lenguas extranjeras, ya que parecen perder de vista que las formas de relacionarse contextualizadas aportan una carga importante a la experiencia epistemológica del nuevo profesor.

Frente a los aspectos externos, con recurrencia notable aparece la metáfora del "abrir puertas". Es decir, la posibilidad de negociar sentidos para la apertura de oportunidades de fortalecimiento con las consecuentes expectativas laborales futuras, ya sea en el mismo sitio de la práctica o en otros: "El colegio [nombre de colegio] me abrió las puertas para ser parte del mismo y dejar una huella en los estudiantes de primaria" (I-0402011-1). La inmersión en la cultura escolar también genera posturas de resistencia frente a discursos instalados que riñen con la epistemología personal o saber propio que empieza a constituirse en los haceres $y$ formas de relacionarse del nuevo profesor de lenguas extranjeras: "no comparto muchas cosas de la filosofía de la institución los cuales [sic] en ocasiones modificaban mi comportamiento en el aula y en la misma institución, el hecho de escuchar a los docentes decir que no hay esperanza con los muchachos, desanima mucho" (I-043-2011-II). Finalmente, se puso de manifiesto y de manera recurrente el adoctrinamiento del cuerpo a las representaciones normalizadas del mismo como un saber, una forma de hacer y de relacionarse fijos de la cultura escolar: "Además, siempre me esmeré por estar bien arreglado" (I-037-2011-I).

\section{Rasgos sociales interpersonales, intrapersonales y externos en el seminario}

Es importante el reconocimiento que se presenta en el campo identitario de los profesores en formación de lenguas extranjeras; ellos son eso: profesores en formación, ya que, en las palabras de uno de los participantes; "Respeté el proceso de cada uno de mis compañeros, pues todos estamos en las mismas condiciones" (II-032-2010-II). El estar en las mismas condiciones, por una parte, podría expresar una postura epistemológica en la cual todos tienen acceso a un repertorio común de conocimiento o lo están elaborando. Esto se manifestó en acciones de apoyo y de cooperación. Es decir, en el seminario se establecieron formas de hacer comunes atravesadas por valores como el respeto (II-032-2010-II; II-02-2010-II; II-011-2010-II) y 
también por disposiciones para escuchar e incorporar retroalimentación de manera informada; así se refleja en las palabras de otro participante: "Tengo una buena relación dialógica con ambos (profesor del seminario y guía pedagógico) lo que me permitió entender y aceptar la retroalimentación y trabajar en esos aspectos para mejorar" (II-08-2012-I).

Por otra parte, en lo que se refiere a las relaciones grupales, dichas condiciones también podrían señalar una forma de simetría basada en principios de solidaridad: "Considero que en el seminario mi participación fue acertada y apreciada por los compañeros, en tanto siempre estuve dispuesta a dar mi opinión que podría aportarle a la discusión de la clase" (II-023-2011-II). Esto generaba autoposicionamientos de prestigio al autoevaluar los aportes realizados como relevantes (II-017-2011-ı) y también al señalar la lucha por la participación y por mantener su calidad (II-0142009-II). El seminario en sí mismo se percibió de manera contradictoria; mientras que para algunos fue el espacio de la discusión, como ya se ha ejemplificado (II-029-2010-॥; ॥-035-2010-ı), para otros implicó un grado de desaprobación: "Desde mi punto de vista el seminario fue un poco denso" (II-044-2009-II). Es notable que no se presentaron en el corpus de datos alusiones a la expectativa laboral (II-041), la presentación personal (II-038), la realización de acuerdos (II-026), el involucramiento (II-020) y la capacidad de adaptación (II-05). Sin embargo, de estos rasgos se pueden argumentar huellas o elementos de presencia en los demás rasgos documentados.

\section{Rasgos sociales interpersonales, intrapersonales y externos relacionados con el guía pedagógico}

Si bien es cierto que durante la práctica pedagógica de los nuevos profesores de lenguas extranjeras existen tensiones manifiestas en la desaprobación (III-045-2009-II) debido a la logística misma del proceso de retroalimentación, es decir, no se comparten los haceres de todos los actores y esto puede tener consecuencias en los repertorios de conocimiento comunes, también hay un predominio de eventos narrativos positivos. Por ejemplo, se observa la potencialidad de inserción en comunidades laborales en la relación profesor practicante-guía pedagógico: “... el acompañamiento es bueno en el sentido de que otra persona que da una retroalimentación para futuros trabajos" (III-042-2010-I).

Dicho acompañamiento requiere reflexión (III-036-2009-II). El acompañamiento visto así conlleva reflexión sobre las acciones que repercuten en el saber de los profesores en formación, por ejemplo, un participante señaló al respecto: "Durante las actividades realizadas en el colegio procuré integrar las observaciones y sugerencias dadas por mi guía pedagógica y reflexionar sobre mi desempeño y los resultados al hacerlo" (III-036-2009-II). 
Frente a los saberes, estos se constituyen de manera dialógica para algunos casos en los cuales el conocimiento es negociado "considero que también aporté algunos conocimientos pedagógicos didácticos a mi guía, los cuales plantearon discusiones muy productivas a nivel profesional" (III030-2012-I). Tanto saberes como haceres y formas de relacionarse tienden a ser prácticas negociadas desde la mirada del otro que involucra lo emotivo (III-024-2011-II) y el acogimiento de la retroalimentación (III-015-2012-II), desde el logro de acuerdos (III-027-2011-I; II-018-2010-II; II-03-2011-ו), desde la consideración y el reconocimiento de factores humanos y sus fortalezas (III-033-2010-I) y desde la comunicación (III-06-2012-I; III-09-2009-I). $\mathrm{Al}$ igual que en el caso del seminario, rasgos como el involucramiento (III-021) y la presentación personal (III-039) no se manifestaron en citas directas dentro del corpus de datos; pero se pueden argumentar huellas o elementos de presencia de estos rasgos en los demás rasgos documentados.

\section{Discusión}

De manera complementaria a la literatura especializada en práctica pedagógica de profesores de lenguas extranjeras (Cote, 2012; Insuasty y Zambrano, 2014; Prada y Zuleta, 2005; Viáfara, 2005a, 2005b, 2011; Zambrano e Insuasty, 2008, 2009; entre otros), este estudio propone considerar las arquitecturas de práctica (Kemmis et al., 2014) que ocurren en la práctica pedagógica como un tejido (de práctica) en el que se intersecan modos de saber, de hacer y de relacionarse.

Pensar la práctica pedagógica de estos nuevos profesores de lenguas extranjeras de esta manera podría rendir más frutos para comprenderla como un espacio social multidimensional y no meramente como un momento en el que ciertos saberes aprehendidos o construidos durante una etapa de estudio simplemente se traducen en haceres en un contexto que suele vincularse a la reflexión como un mecanismo para el mejoramiento del hacer. Es importante (re)imaginar la práctica docente y para ello es necesario comprenderla de manera no tan canónica. No se trata tan solo de un "poner en práctica" a secas. Se trata más bien de realizar en la práctica ciertos modos, los cuales para el caso ilustrado implican por ejemplo una dimensión social que se interseca con otras dimensiones (ej.; lingüística, pedagógica, cognitiva-emocional).

En sí misma esta dimensión social constituye un orden, como lo afirmamos, atravesado por el sentido de estar juntos en cuanto vínculo. A manera de resumen, en la tabla 2 se sintetiza un orden social encontrado en las autovaloraciones de los participantes, manifiesto en eventos narrativos, que interpreta una arquitectura de práctica docente centrada en la dimensión social. 
Tabla 2. Dimensión social de una arquitectura de práctica pedagógica de profesores en formación de lenguas extranjeras

\begin{tabular}{|c|c|c|c|}
\hline Contexto & Modos de saber & Modos de hacer & Modos de relacionarse \\
\hline$\frac{.0}{\frac{O}{0}}$ & $\begin{array}{l}\text { Hay conciencia sobre la } \\
\text { reformulación de ideas } \\
\text { y la modificación de } \\
\text { fórmulas de enseñanza. } \\
\text { Se reconoce la } \\
\text { diversidad y existe una } \\
\text { visión del conocimiento } \\
\text { co-construido. }\end{array}$ & $\begin{array}{l}\text { Se observan acciones } \\
\text { en las que el otro es un } \\
\text { recurso de conocimiento } \\
\text { y de validación de las } \\
\text { acciones; en ese sentido, } \\
\text { el manejo de la clase y } \\
\text { el diseño de materiales } \\
\text { se convierten en modos } \\
\text { de acción que posibilitan } \\
\text { posicionamientos } \\
\text { específicos en los } \\
\text { cuales la reflexión se } \\
\text { convierte en un factor } \\
\text { para tomar decisiones } \\
\text { o cursos de acción de } \\
\text { manera informada. }\end{array}$ & $\begin{array}{l}\text { Desde lo relacional, } \\
\text { hay posicionamientos } \\
\text { interactivos y reflexivos } \\
\text { al construirse } \\
\text { relaciones simétricas y } \\
\text { asimétricas que inciden } \\
\text { en construcciones } \\
\text { identitarias. }\end{array}$ \\
\hline 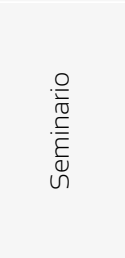 & $\begin{array}{l}\text { Hay conciencia sobre } \\
\text { formas de entendimiento } \\
\text { frente a la emergencia de } \\
\text { disensos y consensos, } \\
\text { dada la deliberación en } \\
\text { torno al conocimiento } \\
\text { y la experiencia. }\end{array}$ & $\begin{array}{l}\text { Con acciones de } \\
\text { cooperación, se busca } \\
\text { hacer conscientes las } \\
\text { experiencias, aunque } \\
\text { también se dan luchas } \\
\text { por el posicionamiento. }\end{array}$ & $\begin{array}{l}\text { Desde lo relacional, el } \\
\text { seminario se convierte } \\
\text { en un espacio para } \\
\text { la construcción del } \\
\text { respeto por el otro y } \\
\text { lo democrático, lo cual } \\
\text { propicia potencialmente } \\
\text { la cohesión grupal. }\end{array}$ \\
\hline 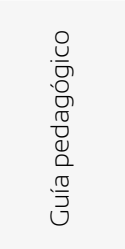 & $\begin{array}{l}\text { Se percibe conciencia } \\
\text { sobre la construcción de } \\
\text { habilidades pedagógicas } \\
\text { y didácticas, y } \\
\text { comprensión de que } \\
\text { es posible compartir } \\
\text { saberes con el otro, así } \\
\text { como diferir de ellos. }\end{array}$ & $\begin{array}{l}\text { Se realizan acciones } \\
\text { para compartir las } \\
\text { relaciones profesionales } \\
\text { y cumplir metas } \\
\text { pedagógicas comunes. }\end{array}$ & $\begin{array}{l}\text { El guía pedagógico se } \\
\text { convierte en el puente } \\
\text { para compartir modos } \\
\text { de relacionarse con el } \\
\text { otro y para establecer } \\
\text { relaciones con miras al } \\
\text { logro de metas comunes. }\end{array}$ \\
\hline
\end{tabular}

Fuente: tomado de Castañeda-Peña et al. (2016a, p. 129).

Las prácticas pedagógicas entonces se estructuran como una arquitectura en la cual los modos de saber son (co)construidos (colegio) y están permeados por emociones, disensos y consensos fundamentados en la deliberación desde experiencias empíricas e informadas (seminario) y en la conciencia sobre el desarrollo de conocimientos tanto pedagógicos como didácticos (guía pedagógico). Nótese que esta comprensión de saber desafía nociones normalizadas sobre el mismo en relación con la formación inicial de docentes, ya que el conocimiento, en una estructura de arquitectura como esta y bajo su dimensión social, es diferenciado y diverso. En ese sentido nos gustaría contribuir con una nominación en plural que reconozca "modos de saberes". Epistemológicamente esto implica que los profesores construyen marcos de interpretación propios que necesitan 
de reconocimiento en la literatura. Parece importante recomendar a los interesados en el tema que observen el conocimiento emocional como parte de los modos de saberes que pueden emerger durante la práctica pedagógica de profesores en formación de lenguas extranjeras.

Los modos de hacer, que también pueden pluralizarse a "haceres", comportan la reflexión como ha sido reconocida en la literatura especializada, en aspectos tanto pedagógicos como didácticos. Pero en las arquitecturas podría tenerse en cuenta la concientización crítica de la experiencia vivida mediante actividades de cooperación en las cuales es innegable la presencia del poder y la emergencia del posicionamiento iterativo. Es decir, el profesor novicio se posiciona mediante sus acciones, pero también es posicionado socialmente. Este posicionamiento se vehicula a través de la mirada del otro y de sus disposiciones para enfrentar mediante "haceres" contextos diversos de enseñanza, como ejercicio de su capital humano (Hargreaves y Fullan, 2012), en el cual el aprendizaje de los idiomas extranjeros de sus estudiantes y los propios adquieren ese mismo carácter: el de diverso. Recientemente, y en conexión con los "modos de saberes", Johnson y Golombek (2016) nos recuerdan la responsabilidad de los formadores de docentes al decir que no es solo en la práctica pedagógica en donde se educa al profesor en formación, sino que es un asunto del currículo, en donde todas las asignaturas son "zonas seguras" que ofrecen "espacios de mediación variados y de múltiples estructuras para que la mediación sensible surja dentro de la formación de los profesores de lenguas extranjeras" (Johnson y Golombek, 2016, p. 132). Esto potencialmente forma parte de la arquitectura de los modos de relacionarse.

En estos modos, se analizó para la presente arquitectura de práctica que la constitución de relaciones conlleva también la de modos simétricos y asimétricos que inciden en el posicionamiento de los actores y de sus identidades sociales en el marco de la práctica pedagógica. Los posicionamientos marcan cohesión (social) en la micropolítica de dicha práctica sobre la cual ejerce gran influencia el guía pedagógico. Es claro que la práctica pedagógica como arquitectura no es un logro individual, sino que es contingente al colectivo y a sus diversos "modos" situados que coadyuvan al logro de capital social del profesor en formación de lenguas extranjeras, el cual comporta

[...] la comprensión de cómo aprenden los niños, la comprensión de su diversidad y de sus circunstancias familiares, el habituarse a la posibilidad de generar prácticas exitosas e innovadoras, y el tener las capacidades emocionales para tener empatía con grupos diversos de estudiantes y de adultos que están en la institución educativa. (Hargreaves y Fullan, 2012, p. 89). 


\section{Conclusiones}

La práctica pedagógica de profesores en formación de lenguas extranjeras se ha venido consolidando en algunas esferas como un espacio para la reflexión con miras al mejoramiento en el actuar en clase. De manera complementaria a esta postura, argumentamos que es necesario comprender la práctica pedagógica como una arquitectura en la que intervienen de forma intrincada modos de saberes, haceres y de relacionarse.

Las situaciones de práctica pedagógica hechas conscientes en eventos narrativos, mediante un formato de autovaloración, por los participantes de las diferentes cohortes de este estudio no se pueden comparar a las vividas y por vivir por otros futuros profesores de lenguas extranjeras en otros contextos de formación y de práctica. Y aunque no es nuestra intención hacer generalizaciones sobre "la arquitectura de práctica", sí creemos que continuar explorando las arquitecturas situadas nos va a permitir comprender desde una voz más local lo que allí sucede y se experimenta. Aquí ilustramos una arquitectura desde una dimensión social. Creemos que esto puede ser parte de un programa de investigación novedoso en el área en el que se intente comprender este fenómeno desde múltiples dimensiones, niveles educativos y contextos.

\section{Referencias}

Castañeda-Peña, H., Rodríguez-Uribe, M; Salazar-Sierra, A. y Chala-Bejarano, P. (2016a). Eventos narrativos, comunidades y arquitecturas de práctica docente: Formación inicial de profesores de inglés y francés. Bogotá: Editorial Universidad Distrital Francisco José de Caldas.

Castañeda-Peña, H., Rodríguez-Uribe, M; Salazar-Sierra, A. y Chala-Bejarano, P. (2016b). Narrative events of pre-service teachers at the end of their teaching practicum with regard to their pedagogical advisor: Learnings reported. Signo y Pensamiento, 68(35): 52-64. DOI:10.11144/Javeriana.syp35-68.neps

Chambers, N. y Jurafsky, D. (2008). Unsupervised learning of narrative events chains. Proceedings of ACL 08: HTL, Association for Computational Linguistics, 789-797.

Cote, G. (2012). The role of reflection during the first teaching experience of foreign language pre-service teachers: An exploratory-case study. Colombian Applied Linguistics Journal, 14(2), 24-34.

Griffin, L. (1993). Narrative, event-structure analysis, and causal interpretation in historical sociology. American Journal of Sociology, 98(5), 1094-1133. 
Hargreaves, A. y Fullan, M. (2012). Professional capital: Transforming teaching in every school. Nueva York: Teachers College en asocio con Ontario Principals' Council.

Insuasty, E. y Zambrano, L. (2014). Experiencias reflexivas de asesoría en la práctica docente. Lenguaje, 42(2), 389-414.

Johnson, K. y Golombek, P. (2016). Mindful L2 teacher education: A sociocultural perspective on cultivating teachers' professional development. Nueva York: Routledge.

Kemmis, S., Wilkinson, J., Edwards-Groves, C., Hardy, I., Grootenboer, P. y Bristol, L. (2014). Changing practices, changing education. Singapur: Springer Science \& Business Media.

Porter, M., Larson, D., Harthcock, A. y Kelly, B. (2003). Re(de)fining narrative events. Journal of Popular Film and Television, 30(1), 23-30.

Prada, L. y Zuleta, X. (2005). Tasting teaching flavors: A group of student-teachers' experience in their practicum. Profile Issues in Teachers' Professional Development, 6, 157-170.

Richards, J. y Nunan, D. (1990). Second language teacher education. Cambridge: Cambridge University Press.

Rowsell, J. y Walsh, M. (2015). Repensar la lectoescritura para nuevos tiempos: multimodalidad, multiliteracidades y nuevas alfabetizaciones. Enunciación, 20(1), 141-150.

Schatzki, Th. (2002). The site of the social: A philosophical account of the constitution of social life and change. University Park, PA: The Pennsylvania State University Press.

Sharky, J. (2009). Can we praxize second language teacher education? A collective, collaborative challenge. Íkala, Revista de Lenguaje y Cultura, 14(22), 125-150.

Strauss, A. y Corbin, J. (2002). Bases de la investigación cualitativa. Técnicas y procedimientos para desarrollar la teoría fundamentada. Medellín: Contus.

Viáfara, J. (2005a). The design of reflective tasks for the preparation of student teachers. Colombian Applied Linguistics Journal, 7, 53-74.

Viáfara, J. (2005b). Responding to observation: How student teachers' use of their counselors' notes support their preparation. Profile Issues in Teachers' Professional Development, 6, 141-156.

Viáfara, J. (2011). How do EfL student teachers face the challenge of using L2 in public school classrooms? Profile Issues in Teachers' Professional Development, 13(1), 55-74.

Woodward, T. (1992). Ways of training. Singapur: Longman 
Zambrano, L. e Insuasty, E. (2008). Analysis of the teaching practicum in the light of a reflective teaching approach. Lenguaje, 36(2), 447-471.

Zambrano, L. e Insuasty, E. (2009). Assessing student-teachers' perceptions about the implementation of reflective teaching tooss in the practicum. Hechos y Proyecciones del Lenguaje, 18, 39-52. 